\title{
The Teaching of the Speaking Skill Using the Audio-Oral Method for the Students of Islamic Senior High School of Zainul Hasan Genggong Probolinggo
}

\author{
Edi Kurniawan Farid, Mamluatun Ni'mah, Nur Arifah \\ Universitas Islam Zainul Hasan (UNZAH) Probolinggo \\ edikurniawanfarid@gmail.com
}

Received: 14-11-2020 $\quad$ Revised: 22-12-2020 $\quad$ Accepted: 31-01-2021

\begin{abstract}
The aims of this research are to know the teaching of the speaking skill using the audio-oral method for the students of Islamic Senior High School of Zainul Hasan Genggong Probolinggo and to know its problems. This qualitative research used observation, interview, and documentation to collect data. To analyze the data, this research based on three basic things, data reduction, data presentation, and extrapolation. And, to measure the validity of the data, this research used triangulation. The results of this research are the teaching of the speaking skill using the audio-oral method for the students of Islamic Senior High School of Zainul Hasan Genggong Probolinggo by giving the dialogues, then translates them and represents the pronunciation in the Arabic dialect, which the students hear and then imitate afterwards sometimes the tape recorder used for improving the arabic speaking skill correctly while teaching continued with more exercises in various Arabic dialogues. The problems of the teaching of the speaking skill using the audio-oral method for the students of Islamic Senior High School of Zainul Hasan Genggong Probolinggo consist of three basic problems which are related to the language environment, the local language, the monotonic teaching method and learning media.
\end{abstract}

Keywords: Audio-Oral Method; Speaking Skill; Teaching Method

$$
\begin{aligned}
& \text { ملخصص } \\
& \text { هدف هذا البحث إلى معرفة تعليم مهارة الكالام بالطريقة السمعية الشفوية لطالبات مدرسة زين الحسن الثانوية } \\
& \text { الإسلامية بقنقون بروبولنجو و معرفة مشكلاهَا. استخدم هذا البحث الكيفي الملاحظة و المقابلة و التوثيق لجمع } \\
& \text { البيانات. ولتحليل البيانات اعتمد هذا البحث على ثلاثة أمور أساسية منها تخفيض البيانات، وعرض البيانات، } \\
& \text { و الاستنباط. و.لمقياس صدق البيانات استخدم هذا البحث التثليث. و نتائج هذا البحث هي تعليم مهارة الكلام } \\
& \text { بالطريقة السمعية الشفوية لطالبات مدرسة زين الحسن الثانوية الإسلامية قنقون بروبولنجو من خلال أن تعطي } \\
& \text { المعلمة الحوارات ثم تترجها وتمثل النطق باللهجة العربية وتسمعها الطالبات ثم تتقلد بعدها أحيانا باستخدم } \\
& \text { المسجل الشريط لغرض ارتقاء مهارة الكالام باللغة العربية الصحيحة مع استمرار التعليم بكثرة التدريبات بأنواع } \\
& \text { الحوارات باللهجة العربية. وأما مشكالات تعليم مهارة الكالام بالطريقة السمعية الشفوية لطالبات مدرسة زين } \\
& \text { الحسن الثانوية الإسلامية قنقون بروبولنجو فتتكون من ثلاث مشكالات أساسية وهي ما يتعلق بالبيئة اللغوية، } \\
& \text { و اللغة المحلية، وطريقة التعليم الرتيبة و الوسائل التعليمية. } \\
& \text { الكلمات المفتاحية: مهارة الكالام، السمعية الشفوية، طريقة التعليم }
\end{aligned}
$$

(C) 2021 Edi Kurniawan Farid, Mamluatun Ni'mah, Nur Arifah 
مهارة الكالام هي إحدى مهارات الأربعة تحتل دورا كبيرا في عملية تعليم اللغة العربية، ولأجل أن يكون الطالب ناجحا في درس اللغة العربية لابد أن يتضمن تعليمه القدرة على الكالام. الكالام في اللغة من المهار ات الأساسية التي تمثل غاية من غايات الدراسة اللغوية وإن كان هو نفسه و سيلة لإِتصال مع الآخرين. و كان الكالام و سيلة من و سائل اتصال الإنسان بغيره من أبناء الأمم الأخرى، به بنقل انفعالاته ومشاعره وأفكاره ويقضي حاجاته وغايته. لذلك أن الكالام أكثر الأفعال في حياة الإنسان و تكون دور هاما في المحتمع ويعتبر من أهم المهارات اللغوية العربية الأربعة منها مهارة الكالام. ومن أهم شيء في تعليم الكالام هو الممارسة يعين ممارسة الكلام، لأن مهارة الكلام هي قدرة تعيين الأصوات المنطوقة أو الكلمات التعبير الفكرة من الآراء أو الارادة أو الشعور للمتكلمين. ويستفيد منها الطلاب أن يتصلوا لسانيا صحيحا و طبيعيا باللغة العربية التي يتعلموها قبل. ومع ذلك لا بد المعلم أن يكون هازا للفرص فيسترفد من خبرته، ويمتاح من طبيعة المنهج الذي يتعامل به، و الموقف التعليمية التي يمر بها.

ومن المعلوم، أن طريقة التعليم وجهان بارزان وهما طريقة التعليم ووسيلة التعليم. و كون و سيلة التعليم و سيلة تعليمية مضمون في جزء الطريقة. كإحدى البيئة التعليمية التي نظمها المدرس ولها دور مهم في عملية التعليم، وعرف المدرس الجيد كيف يعلل الطلاب حتى تكون همتهم زائدة لتعلم مادة الدراسة.``من هنا نعرف أن تدريس مهارة الكالام يعتمد كثيرا على الطريقة و المعلم. ولذلك لا بد من الأخذ بيد المتعلم تدريجيا و حسب الخطوات و الطريقة التي تؤدي إلى الكلام الصحيح. الطريقة هي إحدى العناصر الهامة في بناح عملية التعليم.ّم في الواقع، أن بخاح التدريس يعتمد على ثلاثة عوامل: الأول، دروس التحضير الكمال. و الثاني، طرق التدريس، الثالث، قدرة الطلاب على مواجهة الدروس بكل إخلاص وفهمها فهما جيدا.؛ وذهب أيضا محمود إسماعيل صيني في كتابه دراسة في طرائق تعليم

${ }^{1}$ Fadhlan Masykura Setiadi, “Ta'līm Mahārat Al-Kitābah Li Ghairi Al-Nātiqīna Bihā," Ihya Al 'Arabiyah 1, no. 2 (2015): hlm. 23-37.

${ }^{2}$ S Nasution et al., "Berbagai Pendekatan Dalam Proses Belajar Dan Mengajar," Pendidikam 3, no. 1 (2017): 1-62, https://doi.org/10.1017/CBO9781107415324.004.

${ }^{3}$ Mamluatun Ni'mah, "Al-Muqorror Li Ta'līm Al-Kalām Fī Qismi Ta'līm Al-Lughah Al-'Arabiyah Al-Tābi' Li Kulliyat Al-Ādāb Wa Al-Tarbiyah," IJ-ATL (International Journal of Arabic Teaching and Learning) 2, no. 2 (2018): 90-129, https://doi.org/10.33650/ijatl.v2i2.423.

${ }^{4}$ Ahmad Muhammad 'Abd Al-Qādir, Țuruqu Ta'līm Al-Lughah Al-'Arabiyah (al-Qāhirah: Maktabah alQāhirah, 1989). hlm. 122. 
Edi Kurniawan Farid, Mamluatun Ni'mah, Nur Arifah: The Teaching of The Speaking Skill.......

اللغات الأجنبية أن الطريقة هي الخطة العامة لعرض المادة اللغوية بصورة منتظمة، لا تتناقض أجزائها، و تبتين على مدخل معين.

وإذا نرى عدد المتعلمين الذين اهتموا باللغة العربية فعلى مدرس أن يختار و يعين الطريقة أو المدخل أو الطراز المناسبة المطبقة في عملية تدريس اللغة العربية لاها تؤثر في بهاح وتحقيق أهداف تعليم اللغة العربية.' و كان اختيار الطريقة الصحيحة يصبح حلا حسنا في علاج المشكلة باستفادة نتائج البحوث العلمية الجديدة المناسبة. ولذلك فطريقة التدريس تعتبر أمرا ضروريا يحتاج إليها الانسان لحل المشكالات التعليمية. ومن طرق التدريس هي الطريقة السمعية الشفوية. أو ما يسمى بطريقة Audiolingual هذه الطريقة الصوت الكاسيت والفيديو والأفلام و الشرائح وغيرها. وقد تكون باستخدام لغة الأم كوسيلة التعليم.

تشتمل مدرسة زين الحسن الثانوية الإسلامية بقنقون بروبولنجو على الشعبة العديدة منها الشعبة الدينية التي ركز تعليمها في تعليم اللغة العربية و كذلك باستخدام كتاب التراث.^ تتكون هذه الثعبة على أقسام التعليمية المتنوعة، أحدها قسم تعليم اللغة العربية. وإحدى المهارات اللغوية المرجوة تقدرها الطالبات في هذه الشعبة هي مهارة الككام. ` فتلتزم الطالبات تكلم اللغة العربية في كل الأعمال اليومية كأدة الاتصال، و كانت طريقة السمعية الشفوية في عملية تدريس اللغة العربية طريقة غختارة لمعلمة قسم اللغة العربية.

و مما تؤدي إليها المعلمة في استخدام هذه الطريقة هو أن تكون الطالبات قادرات على أن يتكلمن باللغة العربية جيدا باستخدام المفردات العديدة المسموعة بوسيلة المسجل أو الشريطة ليسمعنها و ينالن المفردات الجحديدة كي يستخدمنها في الكلام. إضافة إلى ذلك، كثير من الطالبات يستخدمن اللغة العربية المحلية وليست اللغة الرسمية في الكالام، ولو كانت المعلمة في كل التعليم تحاول على إعطاء المفردات

${ }^{5}$ Maḥmūd Kāmil Al-Nāqah, Ta'līm Al-Lughah Al-'Arabiyah Li Al-Nātiqīin Bi Lughāt Ukhrōo (al-Su'ūdiyah: Jāmi'ah Ummu al-Qurō, 1985). hlm. 149.

${ }^{6}$ Busyairi Madjidi, Metodologi Pengajaran Bahasa Arab (Yogyakarta: Sumbangsih Offset, 1994). hlm. 1.

${ }^{7}$ Ahmad Izzan, Metodologi Pembelajaran Bahasa Arab (Bandung: Humaniora, 2011). hlm. 137.

${ }^{8}$ EK Farid, "Kesalahan-Kesalahan Shorof Pada Tulisan Bahasa Arab Santri Pondok Pesantren Zainul Hasan Genggong” (UIN Sunan Ampel, 2013), 1, http://digilib.uinsby.ac.id/11371.

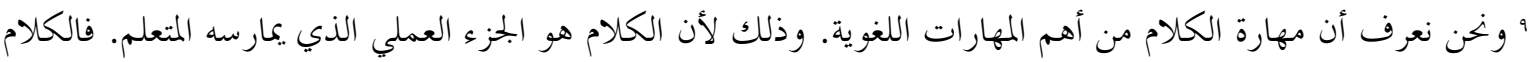
يعبر جزءا أساسيا في تعليم اللغة العربية. 
العلمية والمحادثة. وقلة اهتمام الطلبة بتصحيح كلامهن مع أن المعلمة تقدر على أن تساعدهن فيه. '1 اهتمت الباحثة بهذه المدرسة، لأفا لم تتمكن للعلوم الدينية فقط، ولكن تحتوي على العلوم الحلديثة المختلفة. لذلك تكون هذه الظواهر عجيبة ومهمة خاصة ما يتعلق بتعليم مهارة الكالام باستخدام الطريقة السمعية الشفوية. ولئلا يكون البحث متوسعا إلى ما لا يمتاج إليه، فتحدد هذا البحث إلى هدفين. الأول معرفة تعليم مهارة الككام بالطريقة السمعية الشفوية لطالبات مدرسة زين الحسن الثانوية الإسلامية بقنقون بروبولنجو. و الثاني معرفة مشكالات تعليم مهارة الكاملام باستخدام هذه الطريقة لطالبات مدرسة زين الحسن الثانوية الإسلامية.

استخدم هذا البحث المدخل الكيفي لأن بياناته من نوع البيانات الكيفية التي تصاغ بشكل الكلمات أو الوصف بدلا من الأرقام."' وتتكون البيانات من البيانات الأساسية مثل البيانات عن تعليم مهارة الكالام بالطريقة السمعية الشفوية ومشكلاتها التعليمية، والبيانات الإضافية مثل الوثائق التعليمية و البحوث العلمية. أما أسلوب جمع البيانات فيتكون من الأمرين، الأول ما يتعلق ببيانات عملية التعليم التي حصلت عليها الباحثة من الملاحظة و المقابلة مع المعلمة و ملبرة قسم تعليم اللغة العربية و نائب شؤون الطلاب و نائب برنامج التعليم. و الثاني ما يتعلق ببيانات مشكلات تعليم مهارة الكلام بالطريقة السمعية الثفوية التي حصلت عليها الباحثة من المقابلة مع المعلمة وبعض الطالبات. ووأما تحليل البيانات فقامت الباحثة بالنموذج التفاعلي الذي يتكون من ثلاثة أمور أساسية منها تخفيض البيانات، وعرض البيانات، و الاستنباط. واستخدمت الباحثة تثليثا لمقياس صدق البيانات. قال مولونج التثليث هو إعادة الفحص

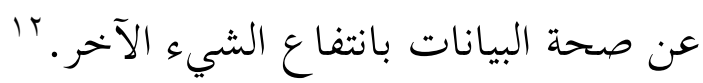

$$
\begin{aligned}
& \text { نتائج البحث و مناقشتها }
\end{aligned}
$$

تعليم اللغة العربية في مدرسة زين الحسن الثانوية الإسلامية قنقون بروبولنجو مدرسة زين الحسن الثانوية الإسلامية قنقون بروبولنجو إحدى المؤسسات التربوية في معهد زين الحسن الإسالامي قنقون. وتشتمل هذه المدرسة على الشعبة العديدة بأقسام التعليمية المتنوعة، منها قسم

•' البيانات التي حصلت عليها الباحثة من المقابلة مع المعلمة اللغة العربية، الأستاذة فطرية، في التاريخ وج من ينايير سنة

${ }^{11}$ Madjidi, Metodologi Pengajaran Bahasa Arab. hlm. 12.

${ }^{12}$ Lexy J Moleong, Metodologi Penelitian Kualitatif (Edisi Revisi) (Bandung: Remaja Rosda Karya, 2017). hlm. 330. 
تحفيظ القرأن وقسم تحقيق الكتب وقسم تعليم اللغة العربية. أما قسم تعليم اللغة العربية فكان موعد أنشطة تنفيذه ليال لكثرة أنشطة التعليم في النهار.

هذا القسم ينفذ في كل ليلة الأربعاء والخميس حول • 11 دقيقة لمحدود وقت التعليم بكثرة برامج التعليم في هذه المدرسة. با "يجري التعليم تحت إشراف المشرفات الثلاثة اللات يساعدن الطالبات في تعلم اللغة العربية من خحلال هذا القسم. ولو كان الإنضمام في هذا القسم ليس من الواجب على جميع الطالبات في هذه المدرسة، ولكن المشاركات لهذا التعليم كثيرة، كلها يتكون من بال طالبة، هن مقسمة إلى قسمين.

أما الكتاب المستخدم لدى المعلمة في التعليم فهو كتاب المحاورة. هذا لأن الكتاب محتمل على أنو اع الحوارات المختلفة كتو جيه تعليم الحوار لترقية مهارة الكلام باستخدام الطريقة السمعية الشفوية تحت إشراف المعلمات.

تعليم مهارة الكالام بالطريقة السمعية الشفوية لطالبات مدرسة زين الحسن الثانوية الإسلامية قنقون بروبولنجو

"يجري التعليم منذ فترة طويلة مع غرض التعليم والخطورات التعليمية المرتبة كإعطاء المفردات وأمر حفظها وتعليم القواعد اللغة العربية كالنحو أو الصرف لقصد ايصال قدرة التكلم باللغة العربية في اليومية. وأما في هذه السنة، تركز مشرفات قسم تعليم اللغة العربية مهارة الكالام. تتكون المشرفات قسم تعليم اللغة العربية من ثلاث مشرفات، واحدة كمدبرة هذا القسم، اسمها أستاذة فطرية، و المشرفتان الباقيتان كمعلمة اللغة العربية اسمها أستاذة نور خالصة باسم معلمة قسم (الألف) تعليم اللغة العربية وأستاذة ألفينا باسم معلمة قسم (الباء) تعليم اللغة العربية.

تكون المدبرة قسم هذا التعليم إلى قسمين يعني قسم (الألف) و(الباء) لكثرة الطالبات المو افقات

هذا التعليم على غرض فعالة أنشطة التعليم حسب قدرات الطالبات. و الحجمم من قدرة الطالبات يركز على تمهيدهن في مهارة الكلام. من لها تمهيد جيد فهي من قسم (الألف) في تعليم اللغة العربية، ومن

rابالبانات التي حصلت عليها الباحثة من المقابلة مع نائب منهج التعليم الأستاذ صالحين، في التاريخ جا 1 من فبرايير سنة 
ها تمهيد مزدجم فهي من قسم (الباء) في تعليم اللغة العربية. وتلك النتائج تدرك من الإمتحان الشفهي في أول خطة إيجاد هذا التعليم. \& تقرر المدبرة لهذا القسم، تعليم مهارة الكالام. لأها من إحدى المهارة المهمة في مهارات اللغة العربية. و كانت طريقة التدريس المختارة والمستخدمة في عملية تعليم مهارة الكاملام لطالبات مدرسة زين الحسن الثانوية الإسلامية هي الطريقة السمعية الشفوية. أما خطو اها فهي أن تعطي المعلمة الحوارات تم تترجمها وتتمثل النطق باللهجة العربية وتسمعها الطالبات ثم تتقلد بعدها أحيانا باستخدم المسجل الثريط لغرض ارتقاء مهارة الكلام باللغة العربية الصحيحة مع استمرار التعليم بكثرة التدريبات بأنواع الحوارات باللهجة العربية. لذا فإها تعتمد التدريبات على الترجمة وإعطاء مادة الحوار العربية كالحوار أو المحادثة بوسيلة كتاب المحاورة أو مسجل الشريط تسمعها الطالبات ثم تقوم بالعملية بعدها. 10 ومن أسباب اختيار هذه الطريقة كما ذكرت الأستاذة لتعويد قدرة الطالبات على تكلم العربية بلهجة صحيح نهو العربي، لأن التراكيب الإندو نيسية تختلف بتراكيب اللغة العربية، والثاني لإدراك الطالبات على التكلم اللغة العربية الصحيحة، تقام هذه الطريقة حتى لا نتكلم العربية إلا صحيحة وفصيحة، لأن لو نتكلم اللغة العربية باللهجة المادوري مثلا، فسماعه غريب وغير لائق من غرض تعليم هذه اللغة. لأن من علامات المهارة من كلام اللغة الأجنبية هي فصاحة الكلام، و كذلك أستخدم كتاب المحاورة في ارتقاء تعليم مهارة الكالام لدى الطالبات كما أن ذلك الكتاب يحتمل عن أنواع الحوارات. الطريقة السمعية الشفوية هي الخطة الشاملة التي يستعين هـا المعلم التحقيق الأهداف المطلوبة من تعلم اللغة خصوصا في ارتقاء الكلام نحو صاحب الكلام المقصودة. 'ا وتتضمن الطريقة ما يتبعه المعلم من أساليب، و إجراءات، وما يستخدمه من مادة تعليمية معينة.

ـ أبيانات التي حصلت عليها الباحثة من المقابلة مع المعلمة اللغة العربية، الأستاذة فطرية، في التاريخخ ب من فبرايير سنة

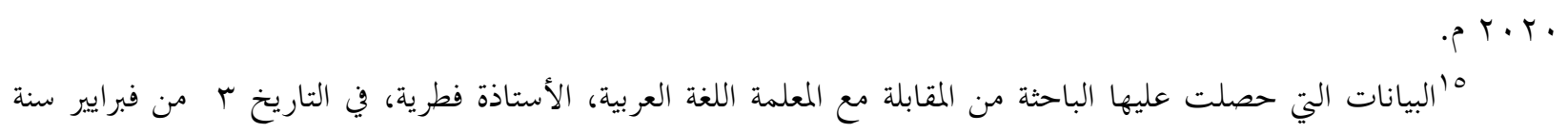
.$r \cdot r \cdot$ 11.

${ }^{16}$ Susan Kifutu, "Background and Characteristics of the Audiolingual Method," TCNJ Magazine, 2015. hlm.

${ }^{17}$ edi Kurniawan Farid, "Tațwīr Al-Mawād Al-Ta'līmiyah Li Mahārah Al-Istimā’ Li Dāris Al-Lughah Al'Arabiyah Li Ghayri Al-Nāțiqīn Bihā,” Alsuna: Journal Of Arabic And English Language 1, no. 2 (2018): 114-20, https://doi.org/https://doi.org/10.31538/alsuna.v1i2.79. 
Edi Kurniawan Farid, Mamluatun Ni'mah, Nur Arifah: The Teaching of The Speaking Skill........

تعليم المهار ات يحتاج إلى العملية، وعملية التعليم تحتاج إلى كثرة التدريبات لإيجاد العادة. و كذلك في تعليم مهارة الكالام، بعد أنشطة الطريقة السمعية الشفوية يحتاج أيضا إلى تدريبات كثيرة نهو تعود

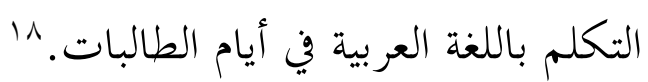
و تستخلص الباحثة أن عملية تعليم مهارة الكلام بالطريقة السمعية الشفوية لطالبات مدرسة زين الحسن

$$
\begin{aligned}
& \text { الثانوية الإسلامية في قسم اللغة العربية هي:19 } \\
& \text { ا ـ مقدمة التعليمية بالسالهام. } \\
& \text { r. تعطى المعلمة مادة الحوار أحيانا بوسيلة المسجل الشريط. } \\
& \text { r. تترجم المعلمة المادة الحوار المقصودة. }
\end{aligned}
$$

ع. تتمثل المعلمة كيفية النطق باللهجة العربية، وتسمع الطالبات المفردات أو العبارات و كذلك كيفية النطق اللغة العربية الصحيحة. ه. تأمر المعلمة أن تقلد الطالبات جماعة أو تحفظها. 7. التدريب على أنماط الكلمات في الحوار. V. تعطي المعلمة المفردات للطالبات ثم تأمرهن أن يكوفا إلى كلمة قصيرة. ^. تعطي المعلمة أنواع الوظيفة كإنشاء العربي أو قصة قصيرة أو نص الخطابة موافقا بقواعد النحو و الصرف و البلاغة لارتقاء جودة مهارقن في تعليم اللغة العربية.

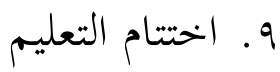

مشكلات تعليم مهارة الكلام بالطريقة السمعية الشفوية لطالبات مدرسة الثانوية الإسلامية زين الحسن قنقون فرو بولنجوا

لكل عملية التعليم توجد المشكات العديدة التي ينبغي للمعلم أن يوجهها ويفكر كيف يحلل هذه المشكالت كي يكون التدريس فعالا ويناسب .ما ترجو المعلمة كي تحقق الأهداف المرجوة. وتوجد

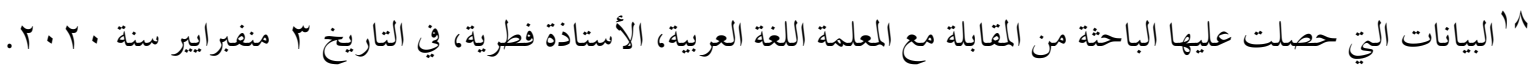

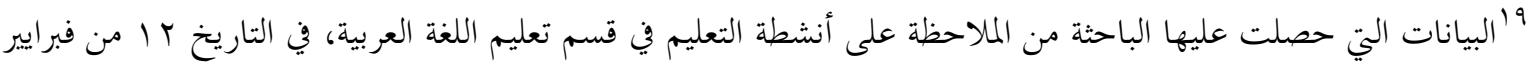


المشكلات في تعليم مهارة الكلام بالطريقة السمعية الشفوية لطالبات مدرسة زين الحسن الثانوية

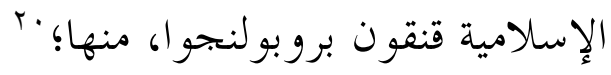

$$
\begin{aligned}
& \text { 1. المشكلة تتعلق بالبيئة }
\end{aligned}
$$

إن تعليم اللغة العربية يحتاج إلى البيئة اللغوية التي تساعد الطالبات في تعويد وممارسة تكلم اللغة

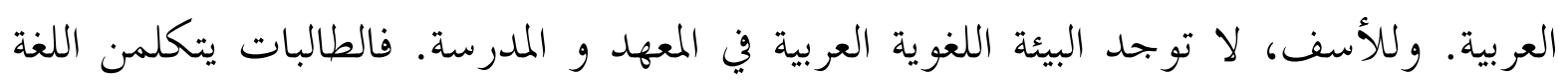

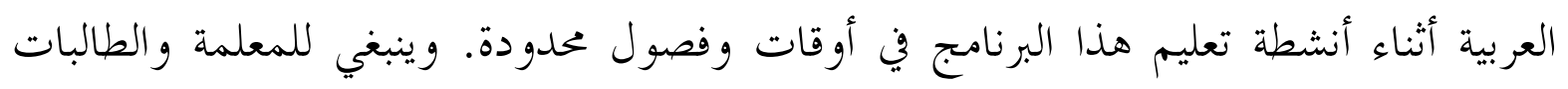
ورئيس المدرسة أن يوفروا أوقات والأماكن تكون فيها البيئة اللغوية ويعتمدوا على النى النظام والقانون

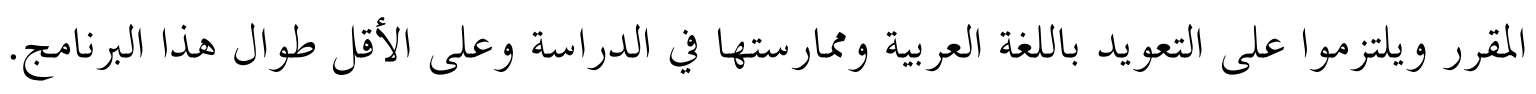
r r المشكلة تتعلق باللغة المحلية

صعبة كيفية نطق الطالبات بلهجة اللغة العربية لإعتاد لغتهن بلهجة اللغة المحلية. وينبغي للمعلمات بلمات

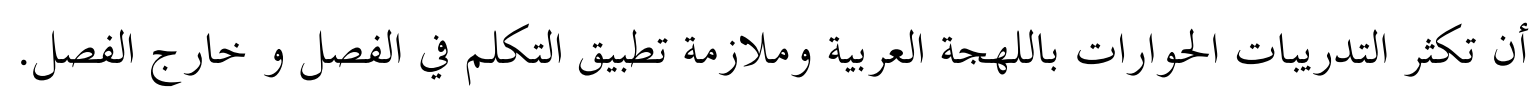
r. المشكلة تتعلق بطريقة التعليم الرتيبة والوسائل التعليمية

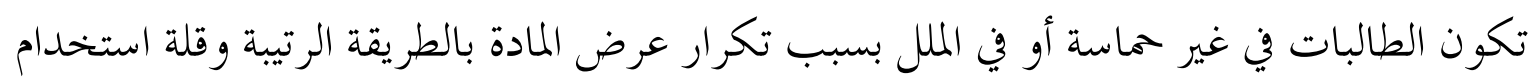

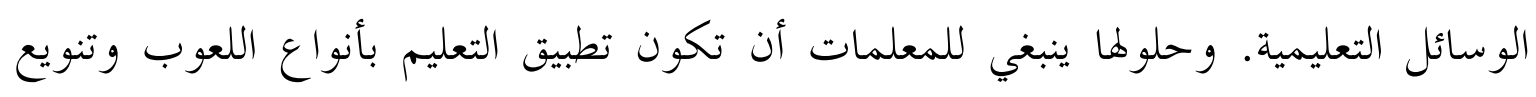

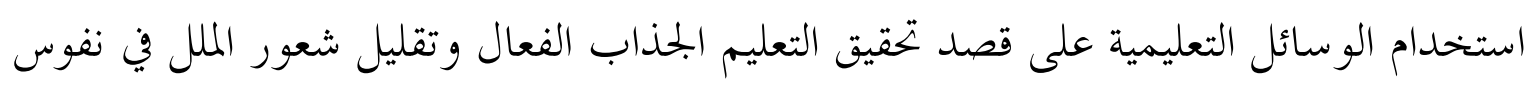
الطالبات.

تم تعليم مهارة الكلام بالطريقة السمعية الشفوية لطالبات مدرسة زين الحسن الثانوية الإسلامية قنقون بروبولنجو من خلال أن تعطي المعلمة الحوارات ثم تترجمها وتتمثل النطق باللهجة العربية

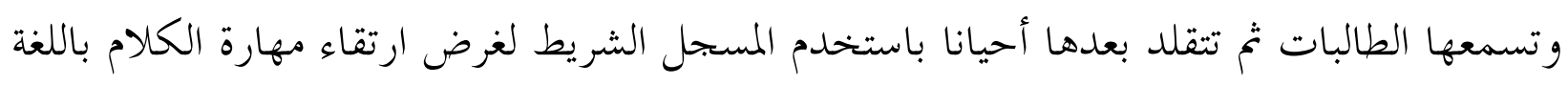

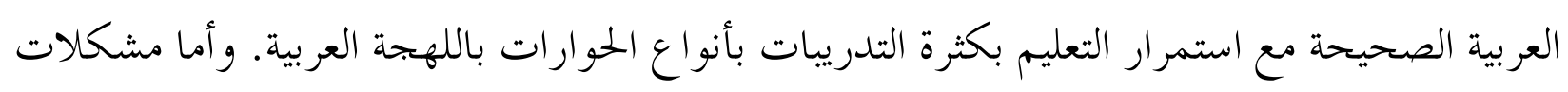
تعليم مهارة الكلام بالطريقة السمعية الشفوية لطالبات مدرسة زين الحسن الثانوية الإسلامية قنقون

•rابليانات التي حصلت عليها الباحثة من المقابلات مع المعلمات قسم تعليم اللغة الغربية، في تاريخ م1 | و 19 من فبرايير سنة $\cdot r \cdot r \cdot$ 
بروبولنجو فتتكون من ثلاث مشكلات أساسية وهي ما يتعلق بالبيئة اللغوية، و اللغة المحلية، وطريقة التعليم الرتيبة و الوسائل التعليمية.

نظرا إلى نتائج هذا البحث هناك بعض التوصيات ينبغي أن يراعيها لجودة تعليم اللغة العربية خاصة ما يتعلق بتعليم مهارة الكام باستخدام الطريقة السمعية الشفوية منها الأولى ينبغي للمعلمة و الطالبات ورئيس المدرسة أن يوفروا أوقات وأماكن تكون فيها البيئة اللغوية ويعتمدوا على النظام و القانون المقرر ويلتزموا على التعويد باللغة العربية وممارستها في الدراسة. و الثانية ينبغي للمعلمات أن تكثر التدريبات الحوارات باللهجة العربية وملازمة تطبيق التكلم في الفصل و خارج الفصل. و الثالثة ينبغي للمعلمات أن تكون تطبيق التعليم بأنواع اللعوب وتنويع استخدام الوسائل التعليمية على قصد تحقيق التعليم الجذاب الفعال وتقليل شعور الملل في نفوس الطالبات.

\section{Bibliografi}

Al-Nāqah, Maḥmūd Kāmil. Ta'lìm Al-Lughah Al-'Arabiyah Li Al-Nātiqiñ Bi Lughāt Ukhrō. al-Su'ūdiyah: Jāmi'ah Ummu al-Qurō, 1985.

Al-Qādir, Aḥmad Muḥammad 'Abd. Țuruqu Ta'lìm Al-Lughah Al-'Arabiyah. al-Qāhirah: Maktabah alQāhirah, 1989.

Farid, Edi Kurniawan. "Tatwūr Al-Mawād Al-Ta’limizah Li Mahārah Al-Istimä’ Li Dāris Al-Lughah Al'Arabiyah Li Ghayri Al-Nätiqin Bihä." ALSUNA: Journal Of Arabic And English Language 1, no. 2 (2018): 114-20. https://doi.org/https://doi.org/10.31538/alsuna.v1i2.79.

Farid, EK. "Kesalahan-Kesalahan Shorof Pada Tulisan Bahasa Arab Santri Pondok Pesantren Zainul Hasan Genggong." UIN Sunan Ampel, 2013. http://digilib.uinsby.ac.id/11371.

Izzan, Ahmad. Metodologi Pembelajaran Bahasa Arab. Bandung: Humaniora, 2011.

Kifutu, Susan. "Background and Characteristics of the Audiolingual Method." TCNJ Magazine, 2015.

Madjidi, Busyairi. Metodologi Pengajaran Bahasa Arab. Yogyakarta: Sumbangsih Offset, 1994.

Moleong, Lexy J. Metodologi Penelitian Kualitatif (Edisi Revisi). Bandung: Remaja Rosda Karya, 2017.

Nasution, S, Hendri Afrianto, Safei \& Jamilah Nurfadillah Salam, Nama Nim, Ida Malati Sadjati, Sebagai Gelling Agent, Terhadap Sifat, et al. "Berbagai Pendekatan Dalam Proses Belajar Dan Mengajar.” Pendidikam 3, no. 1 (2017): 1-62. https://doi.org/10.1017/CBO9781107415324.004.

Ni'mah, Mamluatun. "Al-Muqorror Li Ta'lim Al-Kalām Fì Qismi Ta'lìm Al-Lughah Al-'Arabiyah Al-Täbi' Li Kulliyat Al-Ādäb Wa Al-Tarbiyah." IJ-ATL (International Journal of Arabic Teaching and Learning) 2, no. 2 (2018): 90-129. https://doi.org/10.33650/ijatl.v2i2.423.

Setiadi, Fadhlan Masykura. "Ta'lìm Mahārat Al-Kitäbah Li Ghairi Al-Nätiqina Bỉhä.” Ihya Al'Arabiyah 1, no. 2 (2015): 123-37. 
HALAMAN INI SENGAJA DIKOSONGKAN 